\title{
The long-term effects of space weather on satellite operations
}

\author{
D. T. Welling \\ University of Michigan, Ann Arbor, Michigan, USA \\ now at: Los Alamos National Laboratory, P.O. Box 1663, MS:D466, Los Alamos, NM 87545, USA \\ Received: 24 March 2009 - Revised: 15 June 2010 - Accepted: 16 June 2010 - Published: 24 June 2010
}

\begin{abstract}
Integrated lifetime radiation damage may cause spacecraft to become more susceptible to operational anomalies by changing material characteristics of electronic components. This study demonstrates and quantifies the impact of these effects by examining the National Oceanic and Atmospheric Administration (NOAA) National Geophysical Data Center (NGDC) satellite anomaly database. Energetic particle data from the Geostationary Operational Environmental Satellites (GOES) is used to construct the total lifetime particle exposure a satellite has received at the epoch of an anomaly. These values are compared to the satellite's chronological age and the average exposure per year (calculated over two solar cycles.) The results show that many anomalies occur on satellites that have received a total lifetime high-energy particle exposure that is disproportionate to their age. In particular, $10.8 \%$ of all events occurred on satellites that received over two times more 20 to $40 \mathrm{MeV}$ proton lifetime particle exposure than predicted using an average annual mean. This number inflates to $35.2 \%$ for 40 to $80 \mathrm{MeV}$ protons and $33.7 \%$ for $\geq 2 \mathrm{MeV}$ electrons. Overall, $73.5 \%$ of all anomalies occurred on a spacecraft that had experienced greater than two times the expected particle exposure for one of the eight particle populations used in this study. Simplistically, this means that the long term radiation background exposure matters, and that if the background radiation is elevated during the satellite's lifetime, the satellite is likely to experience more anomalies than satellites that have not been exposed to the elevated environment.
\end{abstract}

Keywords. Interplanetary physics (Energetic particles) Magnetospheric physics (General or miscellaneous)

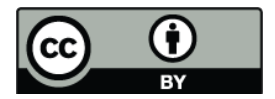

Correspondence to: D. T. Welling (dwelling@lanl.gov)

\section{Introduction}

Anomalous satellite behavior can disrupt spacecraft operation, negatively affecting all who rely on space borne assets - from operators and service providers to consumers. While there are many causes of these problems, such as software glitches or hardware failure, many can be induced by the effects of the space environment (Baker, 2000; Feynman and Gabriel, 2000; Koons et al., 1999; Pirjola et al., 2005). Satellite engineers and operators work diligently to prevent the occurrence of spacecraft anomalies, but space weather introduces an uncontrollable, hard to predict variable into the system.

There is a growing body of work that correlates the occurrence of these anomalies with space weather conditions. Krause et al. (2000) found significant correlations between the geomagnetic activity indices $\mathrm{Kp}$ and Dst and observed surface charging values from the Defense Satellite and Communication System III spacecraft. Fennell et al. (2001) found similar correlations between these indices and the SCATHA (Spacecraft Charging AT High Altitudes) satellite, noting that surface charging events correlated to substorm activity while internal charging events corresponded to geomagnetic storm events. Wilkinson et al. (1991) showed that single event upset (SEU) anomalies recorded by the TDRS1 (Tracking and Data Relay Satellite) were associated with increases in galactic cosmic ray and high energy proton fluxes. Both Iucci et al. (2005) and Pilipenko et al. (2006) used the National Oceanic and Atmospheric Administration's (NOAA) National Geophysical Data Center's (NGDC) spacecraft anomaly database to show that on days when there was an increase in anomalies, there was a corresponding increase in in-situ particle flux measurements and/or geomagnetic indices. While all of these studies illustrate the connection between space weather conditions at the time of the anomaly and the spacecraft disruption itself, they all neglect the long term effects of the background radiation environment. 


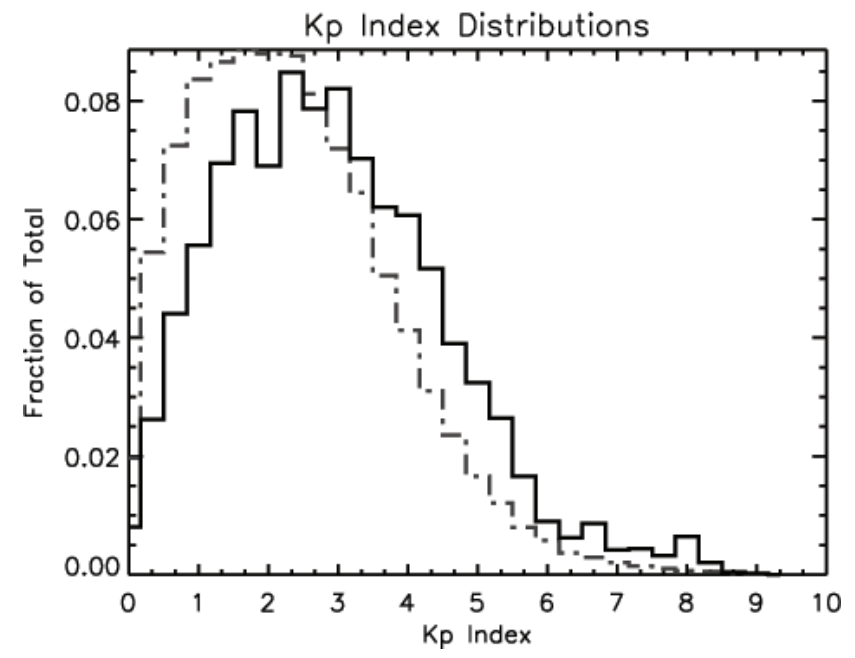

Fig. 1. Distribution of $\mathrm{Kp}$ index values corresponding to NGDC database anomaly epochs (solid line) and the distribution of all $\mathrm{Kp}$ values over the temporal span of the database (1963 to 1994). Although there is a clear shift between the two distributions, only $15 \%$ of all anomalies in the database occured during disturbed or stormier (greater than $5^{-} \mathrm{Kp}$ ) conditions.

Energy deposited onto the spacecraft from high energy particles and electromagnetic radiation has the ability to break down and rearrange atomic bonds of spacecraft materials, from paints and coatings to electronic insulators, dielectrics and circuitry components. These total dose effects are described by Scarpulla and Yarbrough (2003, and references therein). Over time, coatings become less reflective and electronics change their characteristics and this may cause the satellite to become more likely to experience an operational anomaly. Investigating solar and magnetospheric activity during or near the epoch of the anomaly will not uncover correlations between space weather and anomalies driven by long term radiation damage.

Total dose damage and its effects present a special problem for satellite operators and the space research community. Without the proper instrumentation on board, it is difficult to know how much particle radiation exposure a particular satellite has experienced (described as a satellite's "radiation life" by Gubby and Evans (2002).) Additionally, total exposure effects are often confused with normal satellite aging due to insufficient diagnostics (Dyer, 2002). These complications have made understanding the full importance of these effects especially challenging. This study explores the relationship between long-term radiation exposure and spacecraft operational anomalies by investigating the total life time high energy particle exposure experienced by a spacecraft at the time an anomaly occurred.
Table 1. Analysis of perceived age distributions. The left column lists the eight GOES EPS particle/energy channels used in this study. The second column lists the medians of each distribution. The third column shows the percent of anomalies of all analyzed where the perceived age was greater than the actual age at the time of the anomaly. Finally, the right-most column reports if the sample represents a parent population where greater than $50 \%$ of all anomalies have perceived ages greater than the actual ages $(99 \%$ confidence level).

\begin{tabular}{lccc}
\hline Particle channel & Median & $\%>$ Age & $\pi_{+}>.5 ?$ \\
\hline$\geq 2 \mathrm{MeV}$ electrons & 2.511 & $55.7 \%$ & YES \\
0.8 to $4 \mathrm{MeV}$ protons & 1.106 & $08.6 \%$ & No \\
4 to $9 \mathrm{MeV}$ protons & 1.084 & $41.3 \%$ & No \\
9 to $15 \mathrm{MeV}$ protons & 1.225 & $43.9 \%$ & No \\
15 to $40 \mathrm{MeV}$ protons & 1.785 & $53.0 \%$ & YES \\
40 to $80 \mathrm{MeV}$ protons & 2.003 & $58.4 \%$ & YES \\
80 to $165 \mathrm{MeV}$ protons & 0.459 & $03.2 \%$ & No \\
110 to $500 \mathrm{MeV}$ protons & 0.829 & $07.3 \%$ & No \\
\hline
\end{tabular}

\section{Procedure}

Geostationary Operational Environmental Satellites (GOES) energetic particle flux data was collected from 1974 to 1994. This data consists of an integral measurement of $\geq 2 \mathrm{MeV}$ electrons and a seven channel differential measurement of protons, all recorded by the Energetic Particle Sensor (EPS) instrument aboard the Space Environment Monitor (SEM) subsystem. A list of the proton energy channels is displayed in the first column of Table 1. This data was time-integrated to yield total particle exposure per day. For days where there was more than one GOES satellite present, the multiple measurements were averaged together to create values that better reflect conditions at all geosynchronous locations. These averaged daily particle exposure values were used to investigate satellite anomalies that were reported to the NOAA NGDC spacecraft anomaly database (described in Wilkinson, 1989).

The anomaly database was refined to fit the confines of this study. First, any anomaly for which the launch date of the satellite could not be found was discarded. Launch dates were unattainable for satellites whose real name was not used in the database. Anomaly events that occurred on satellites that were launched before July 1974 were also discarded, because a lifetime exposure could not be calculated using the GOES data. Finally, only anomalies from satellites in geosynchronous orbits were used in order to match the orbit type of the GOES measurements. This reduced the anomaly database to 1609 events from 4996 total.

The NGDC database can be perceived as being limited in scope because anomalies reported to the database managers were, at least initially, thought to be caused by the space environment. However, events were reported on a voluntary basis, often before a formal investigation and without a follow up to improve database accuracy. Additionally, $51 \%$ of all 
anomalies reported to this database are catagorized as having an unknown diagnosis (21\% are diagnosed as surface charging events, $16 \%$ single event upsets, and $9 \%$ internal charging, 3\% other). Figure 1 shows the distribution of the Kp indexes recorded at the time of each spacecraft anomaly. Of all the events reported, only $15 \%$ occurred when the Kp index was $5^{-}$or greater (disturbed magnetospheric conditions or stormier.) Apart from the selection criteria described above, no bias towards anomaly type or diagnosis was shown in this study.

For each anomaly, the lifetime energetic particle exposure was calculated by summing all daily values from the beginning of the satellite's life to the date the anomaly occurred. This value was normalized by dividing by the average particle exposure per year (calculated over two solar cycles). The "exposure-years" experienced by a satellite at the time of the anomaly was compared to the chronological age of the satellite. This process was repeated for all eight particle channels listed in Table 1.

By converting the units of particle exposure to units of time ("exposure-years"), a number is created that reveals how many years worth of typical particle exposure a satellite has experienced. This number, the satellite's "perceived age," can be much different than a satellite's actual age. In addition, the perceived age based on ring current electrons will be different than the perceived age based on galactic cosmic ray protons because of the different processes that govern the separate particle populations.

A useful analogy for understanding this unit is miles on a car. The transmission may fail when the car is only five years old chronologically, but the car perceives that it is ten years old because it has been driven for as many miles as a typical ten year old car (in other words, ten "mile-years"). While miles on a car represents wear and tear from use of the car, the perceived age of a satellite in this study represents wear-and-tear from the effects of particle exposure. It is an approximation of the radiation life referred to by Gubby and Evans (2002).

\section{Results}

Figure 2 visually summarizes the entire data set. The distribution of chronological ages is shown at the bottom, with the perceived ages from the lowest to highest proton channel and finally the electron channel at top. The median of each distribution is shown as the redline bisecting the box and is listed in Table 1; the box boundaries mark the upper and lower quartiles. The whiskers extend from the upper/lower quartile to the value of the quartile plus 1.5 times the interquartile range; green dots mark outliers. In terms of median and spread, the perceived ages calculated from the $\geq 2 \mathrm{MeV}$ electron and $40-80 \mathrm{MeV}$ proton channels stand out compared to the chronological age distribution.

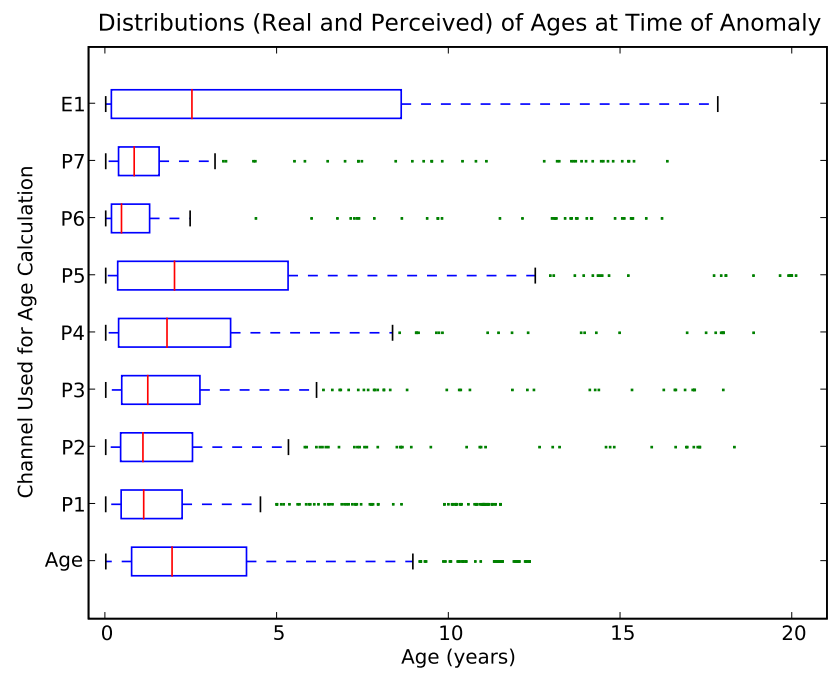

Fig. 2. Box and whisker representation of the distribution of satellite actual ages (bottom box) and perceived ages calculated from each energy channel. P1 marks the lowest proton channel listed in Table 1, P7 the highest, and E1 marks the electron channel.

The top frame of Fig. 3 explores the distribution of satellite chronological age at the epoch of an operational anomaly more closely. Just over $30 \%$ of anomalies occur when the satellite is one year old or less. The distribution drops off quickly, with barely $5 \%$ occurring on satellites that are greater than ten years old.

In the second frame of Fig. 3, the distribution of satellite perceived age based on total 4 to $9 \mathrm{MeV}$ proton exposure at the epoch of an operational anomaly is displayed. Most anomalies occurred on satellites that had only experienced less than two typical years worth of particle exposure for this energy channel. The median of this distribution, 1.084 (listed in the second column of Table 1), is less than that of the median of actual ages, 1.932. Because of this and the distribution's similarities to the distribution of the chronological ages, this data suggests that 4 to $9 \mathrm{MeV}$ protons have little effect on a satellite's susceptibility to anomalies throughout the satellite's life.

For higher energy proton exposure (40 to $80 \mathrm{MeV}$ ), the distribution changes dramatically (Fig. 3, third frame from the top). More satellites have a higher perceived age when an anomaly occurs. Comparing this to the actual age distribution shows that a portion of the anomalies happened on satellites that received a lifetime particle exposure that is disproportionate to that satellite's age. These satellites are wearing down faster than they are aging. This pattern is greatly exacerbated when perceived ages are calculated using the $\geq 2 \mathrm{MeV}$ electron channel (Fig. 3, bottom frame). While only a handful of anomalies occurred on satellites that were $\geq 5$ years old, many anomalies occurred on satellites whose perceived age is $\geq 5$ exposure years. 


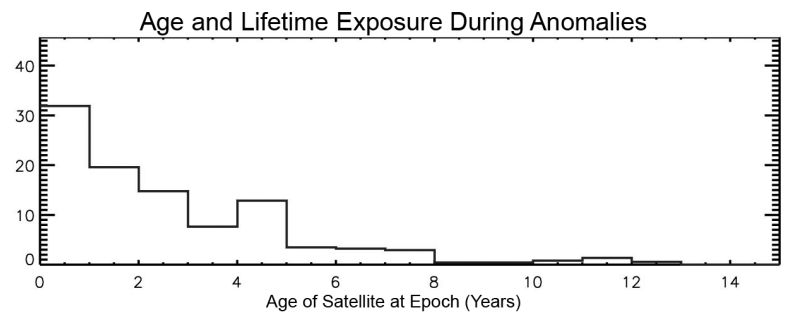

4 - 9 MeV Protons

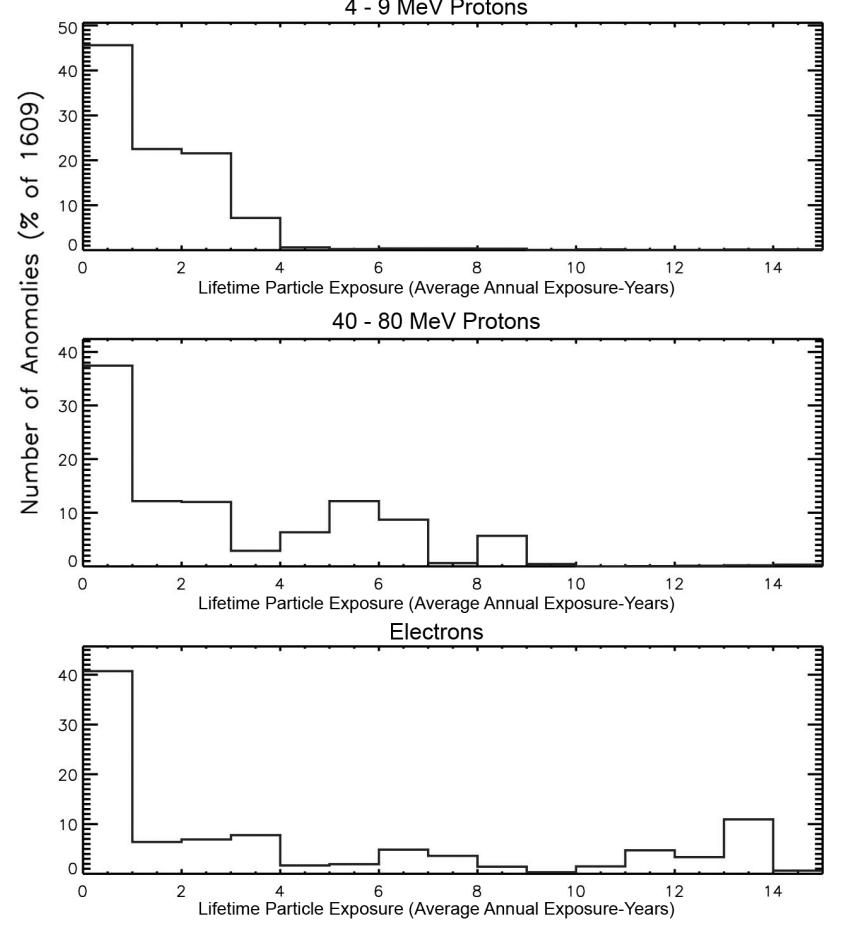

Fig. 3. Distribution of satellite age when an operational anomaly occured (top frame), perceived age calculated from $4-9 \mathrm{MeV}$ protons (2nd frame from top), perceived age calculated from $40-80 \mathrm{MeV}$ protons (3rd frame from top), and perceived age calculated from $\geq 2 \mathrm{MeV}$ electrons (bottom frame).

These patterns can be further demonstrated by examining the proportion of events whose perceived age at the time of the anomaly was greater than the chronological age. The second column of Table 1 shows the proportion of the total number of anomalies for which the perceived age calculated for that particular channel and event is greater than the chronological age of the satellite for the same event (in other words, the satellite perceived that it was older than its actual age based on lifetime particle exposure.) Again, the 15$40 \mathrm{MeV}$ proton, $40-80 \mathrm{MeV}$ proton, and electron channels stand out because for each of these channels, more than $50 \%$ of the events occured when the perceived age outweighed the chronological age.

Statistical inferences can be made about all satellite anomalies by treating the NGDC database as a sample of the larger population. Here, the hypothesis that the anoma-
Table 2. Analysis of perceived to actual age ratios. The left column lists the eight GOES EPS particle/energy channels used in this study. The center column lists the average ratio of perceived age to chronological age for all 1609 anomaly events. The rightmost column lists the percent of the events whose age ratio exceeded two.

\begin{tabular}{lcr}
\hline Particle Channel & Median & $\%>2$ \\
\hline$\geq 2 \mathrm{MeV}$ electrons & 1.339 & $33.7 \%$ \\
0.8 to $4 \mathrm{MeV}$ protons & 0.564 & $<0.5 \%$ \\
4 to $9 \mathrm{MeV}$ protons & 0.825 & $1.1 \%$ \\
9 to $15 \mathrm{MeV}$ protons & 0.908 & $1.0 \%$ \\
15 to $40 \mathrm{MeV}$ protons & 1.170 & $10.8 \%$ \\
40 to $80 \mathrm{MeV}$ protons & 1.215 & $35.2 \%$ \\
80 to $165 \mathrm{MeV}$ protons & 0.244 & $<0.5 \%$ \\
110 to $500 \mathrm{MeV}$ protons & 0.432 & $0.6 \%$ \\
Combined channels & 2.583 & $73.5 \%$ \\
\hline
\end{tabular}

lies analyzed in this work are drawn from a population where most $(>50 \%)$ of the events occur on spacecraft where the perceived age calculated from the different channels is greater than the chronological age is tested. Given the nonnormal, non-symmetric shape of the distributions in question, the binomial sign test (described in detail by Sheskin, 2007, and many others) is an appropriate tool. This test divides each event into two categories: perceived age is greater than the chronological age (a postive result) or not (a negative result). The probability that the sample could have been drawn from a larger population where there is an equally likely chance of a postitive $\left(\pi_{+}\right)$or negative $\left(\pi_{-}\right)$result is calculated. If the calculated probability is greater than $1 \%$, the null hypothesis,

$H_{0}: \pi_{+}=0.5$

cannot be rejected and it is concluded that there is an equally likely chance of a perceived age being greater or less than the chronological age at the time of anomaly occurence. However, if the calculated probability is less than $1 \%$ and there were more positive results than negative, the null hypothesis is rejected and the directional alternative hypothesis,

$H_{A}: \pi_{+}>0.5$

is supported at a $99 \%$ confidence level.

The rightmost column of Table 1 lists whether or not the directional alternative hypothesis is supported with at the $99 \%$ confidence level. Consistent with the qualitative observations above, the $15-40 \mathrm{MeV}$ proton, $40-80 \mathrm{MeV}$ proton, and integrated electron channels stand out. When these channels are used to calculate the perceived age, the majority of the events of the parent population are likely to have greater perceived than chronological ages. Data from the other channels do not support the alternative hypothesis, so no correlation can be drawn between long term damage driven by these particles and anomaly occurrence. 
For each satellite anomaly, the perceived age for each particle channel is divided by the actual age of the satellite. This number indicates how much more or less particle exposure a satellite has received compared to its actual age, or how much faster (or slower) a satellite is wearing down due to high energy particle exposure than it is chronologically aging. This value allows for a quantifiable investigation of the data.

Table 2 shows a summary of this analysis. The center column shows the median perceived to chronological age ratio for each particle channel used in this study. This ratio is less than one for lower energy proton channels, but larger than one for the electron, 15 to $40 \mathrm{MeV}$ proton and 40 to $80 \mathrm{MeV}$ proton channels. The rightmost column lists the fraction of events that occurred on satellites that received more than two times the expected total exposure given their chronological age (age ratio of two or greater.) The $\geq 2 \mathrm{MeV}$ electron, 15 to $40 \mathrm{MeV}$ and 40 to $80 \mathrm{MeV}$ proton channels stand out again with values of $33.7 \%, 10.8 \%$ and $35.2 \%$, respectively.

Further analysis is performed by considering only the maximum perceived age of the eight channels for each anomaly. The resulting distribution is presented in the top frame of Fig. 4. 73.5\% of all anomalies occurred on satellites that are wearing down more than twice as fast as they are chronologically aging due to one of the eight particle populations measured. This distribution may be skewed by anomalies that occurred on very young satellites. It is unlikely that these early anomalies are the result of integrated particle damage. The second frame of Fig. 4 includes only events from satellites that were one year old or older when the anomaly occurred. The number of satellites that are wearing down twice as fast as they are aging increases to $78 \%$. For anomalies that occurred on older (five years or greater, bottom frame of Fig. 4) satellites, this number drops to $50.5 \%$. The number of events and median values for these three distributions is listed in columns two and three, respectively, in Table 3.

The binomial test is again employed, this time to test whether the median of the parent population of maximum perceived/actual age ratios from which the sample shown in Fig. 4 is drawn from is greater than a given value. To do this, the events are divided up into two categories: values that are greater than the test median $\left(\Theta_{\text {test }}\right)$ and values that are less than the test value. Values that are exactly equal to $\Theta_{\text {test }}$ are discarded. The null hypothesis that the population median $\left(\Theta_{\mathrm{p}}\right)$ is equal to $\Theta_{\text {test }}$,

$H_{0}: \Theta_{\mathrm{p}}=\Theta_{\text {test }}$

is tested against the directional alternative hypothesis

$H_{0}: \Theta_{\mathrm{p}}>\Theta_{\text {test }}$

$H_{0}$ is rejected when the calculated probability is less than $1 \% ; H_{A}$ is supported when $H_{0}$ is rejected and there are more anomalies with perceived/actual age ratios greater than $\Theta_{\text {test }}$ than not.
Table 3. Analysis of the distribution of the maxium perceived-toactual age ratios (of the eight calculated for each anomaly) broken down by all anomalies (top row), anomalies occuring on satellites one year of age and older (center row), and anomalies occuring on satellites five years of age and older (bottom row). Number of events, medians, and the percent of the distribution that falls to the right of 2 is shown. The two right-most columns report if the sample represents a parent population whose medians are greater than 2 or 2.5 (99\% confidence level).

\begin{tabular}{lccccc}
\hline Group & $n$ & Median & $\%>2$ & $\Theta>2 ?$ & $\Theta>2.5 ?$ \\
\hline All anomalies & 1609 & 2.583 & $73.5 \%$ & YES & YES \\
Age $\geq$ 1 Year & 1098 & 2.630 & $78.0 \%$ & YES & YES \\
Age $\geq$ 5 Years & 214 & 2.069 & $50.5 \%$ & No & No \\
\hline
\end{tabular}

Table 3 summarizes the results of this test for $\Theta_{\text {test }}=2$ and $\Theta_{\text {test }}=2.5$. For all anomalies in the given sample, $H_{A}$ is supported at the $99 \%$ confidence level for both values of $\Theta_{\text {test }}$. This strongly suggests that in the total population of all satellite anomaly events, over $50 \%$ have a perceived/actual age ratio larger than 2.5 for at least one of the GOES particle channels. When the sample is reduced to satellites with chronological ages $\geq 1, H_{A}$ is still supported at this confidence level. Finally, when the sample is reduced to satellites with chronological ages $\geq 5$, the null hypothesis cannot be rejected.

\section{Discussion and conclusions}

Of the anomalies investigated, the majority occur on satellites that have received a lifetime particle exposure that is disproportionate to that satellite's chronological age. There is a strong likelihood that the sample of anomaly events provided by the NGDC database represents the overall population of operational irregularities. These results show that the historical conditions leading to most anomalies are congruous for making satellites more anomaly prone. The prevalence of the integrated electron channel and the 15 to $80 \mathrm{MeV}$ proton channels in all of the results indicates that the effects of deep dielectric charging and SEUs are probable suspects for causing long term damage. Other particle and energy channels appear uncorrelated to anomaly occurrence.

This pattern becomes less prevalent in older (five years or greater) satellites, as seen in Fig. 4. Gubby and Evans (2002) found that sensitivity to space weather on the Anik E2 satellite decreased with time in orbit, and they speculated that one cause of this may be integrated radiation effects. The results shown in the bottom frame of Fig. 4 support this hypothesis, but there are too few events that fall into this age bracket $(n=214)$ to draw a solid conclusion.

Interpretation of the GOES electron results in the bottom panel of Fig. 3 is not straightforward because the EPS electron detector responds significantly to $>32 \mathrm{MeV}$ protons. 

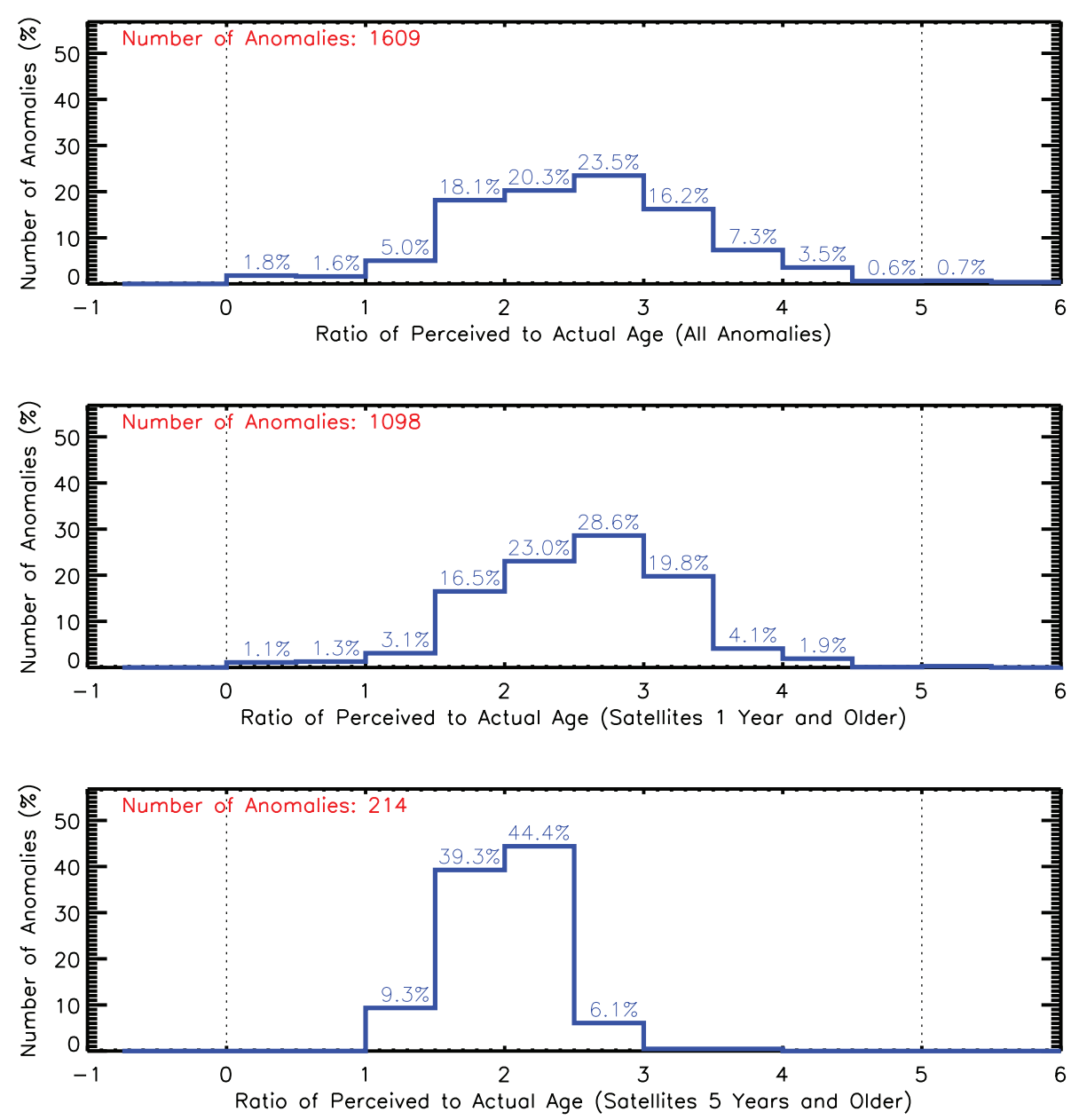

Fig. 4. Distributions of the greatest ratio of perceived age to chronological age. The top frame includes all anomaly events, while the middle frame includes only events where the satellite was one year old or older at the time the event occured, and the bottom frame includes only satelites that were 5 years old and older.

Hence, during periods of increased proton flux, this data becomes polluted. For this reason, the electron results represent the total impact of electrons and protons combined. Isolating the impact of the electrons alone may require another data set.

There are several other limitations that, if properly addressed, could enhance the completeness of this study:

1. Environmental data localized to the spacecraft that experienced the anomaly could be increased. By reducing the flux measurements to a single average value, any spatial variation is lost. The nominal situation would be to have a particle detector on every spacecraft, but this is not feasible.

2. The limitations of the scope of the NGDC anomaly database, as described earlier, is also an issue. It remains unknown if this database is an accurate representation of all anomalous satellite behavior. Recur- ring problems on a single satellite may or may have not been consistently reported, depending on the operator. Obtaining additional events is extremely difficult, however, due to the commercial impacts releasing such data would have on operators (Koons et al., 1999).

3. Other variables, such as micrometeroid and ultraviolet radiation exposure, can play a significant role in satellite performance. These factors were not taken into account in this study, and may be difficult to properly account for. It should be noted that these effects may resonate with the high-energy effects examined here. For example, solar electromagnetic radiation varies with the solar cycle with solar protons, potentially exaggerating the effects of the latter.

Another issue of this study is potential skewing of the results caused by the natural seasonality of space weather. Energetic electron fluxes in the magnetosphere are raised during solar 
minimum periods (Miyoshi et al., 2004); for proton flux variations this pattern is in phase with the solar cycle (Simunac and Armstrong, 2004). Such seasonality may artificially inflate perceived ages calculated here depending on the date of the anomaly. This is especially problematic if there is a similar seasonality observed in event occurrence. Fluctuations of time scale smaller than a year should not impact these results, however, especially for the $>2$ year old satellite results.

To address this, correlation of yearly average perceived ages based on electron and 40 to $80 \mathrm{MeV}$ proton exposure to sunspot number was investigated. Correlation coefficients, designated as " $r$ ", were calculated; a value of $r= \pm 1(0)$ demonstrates perfect (no) correlation or anti-correlation. Both comparisons yielded poor correlation $(r=-0.376$ and 0.277 , respectively). In the case of ions, the correlation coefficient is nearly half of what is found in Simunac and Armstrong (2004). Furthermore, event occurrence correlates extremely poorly $(r=-0.141)$ to the solar cycle. These results imply that seasonality does not play a strong role in this study. To fully eliminate seasonality as a major contributor, however, a complete anomaly database that spans more than two cycles is required.

Despite these limitations, the evidence here strongly suggests that the long-term effects of the background radiation environment indeed play an important role in the occurrence of spacecraft anomalous behavior. There is a strong correlation between enhanced lifetime high energy particle exposure and anomaly occurrence. The potential to create anomalies during unperturbed periods makes the effects of space weather even more difficult to understand and mitigate. Operators, engineers, and scientists must not neglect a spacecraft's integrated particle exposure when investigating the cause of an anomaly.

While predicting and preventing anomalies caused by total dose effects may be difficult, operators can take steps to monitor a spacecraft's perceived age. In situ particle and electromagnetic radiation measurements can yield lifetime exposure estimates. Assimilative and first principle based simulations can complement these measurements by providing better spatial data. By tracking perceived age information, operators can see how fast their equipment is wearing down and know which satellites are more likely to experience problems.

Acknowledgements. The author would like to thank the NOAA's Space Environment Center and National Geophysical Data Center for making their data available. The author is also grateful to Aaron Ridley for his help in preparing this manuscript as well as Steven Morley for the useful discussions concerning statistics.

Topical Editor I. A. Daglis thanks J. L. Roeder and another anonymous referee for their help in evaluating this paper.

\section{References}

Baker, D.: The Occurrence of Operational Anomalies in Spacecraft and Thei Relationship to Space Weather, IEEE Transactions on Plasma Science, 28, 2007-2016, 2000.

Dyer, C.: Radiation effects on spacecraft \& aircraft, in: Solspa 2001, Proceedings of the Second Solar Cycle and Space Weather Euroconference, edited by: Sawaya-Lacoste, H., vol. 477 of ESA Special Publication, pp. 505-512, 2002.

Fennell, J. F., Koons, H. C., Roeder, J. L., and Blake, J. B.: Spacecraft Charging: Observations and Relationship to Satellite Anomalies, in: Spacecraft Charging Technology, edited by: Harris, R. A., vol. 476 of ESA Special Publication, pp. 279, 2001.

Feynman, J. and Gabriel, S. B.: On space weather consequences and predictions, J. Geophys. Res. (Space Physics), 105, 1054310564, doi:10.1029/1999JA000141, 2000.

Gubby, R. and Evans, J.: Space environment effects and satellite design, J. Atmos. Solar-Terr. Phys., 64, 1723-1733, 2002.

Iucci, N., Levitin, A., Belov, A., Eroshenko, E., Ptitsyna, N., Villoresi, G., Chizhenkov, G., Dorman, L., Gromova, L., Parisi, M., Tyasto, M., and Yanke, V.: Space weather conditions and spacecraft anomalies in different orbits, Space Weather, 3, S01001, doi:10.1029/2003SW000056, 2005.

Koons, H. C., Mazur, J. E., Selesnick, R. S., Blake, J. B., and Fennell, J. F.: The Impact of the Space Environment on Space Systems, NASA STI/Recon Technical Report N, pp. 69036, 1999.

Krause, L., Dichter, B., Knipp, D., and Ray, K.: The Relationship Between DSCS III Sunlit Surface Charging and Geomagnetic Activity Indices, IEEE Transactions on Nuclear Science, 47, 2224-2230, 2000.

Miyoshi, Y. S., Jordanova, V. K., Morioka, A., and Evans, D. S.: Solar cycle variations of the electron radiation belts: Observations and radial diffusion simulation, Space Weather, 2, 0, doi: 10.1029/2004SW000070, 2004.

Pilipenko, V., Yagova, N., Romanova, N., and Allen, J.: Statistical relationships between satellite anomalies at geostationary orbit and high-energy particles, Adv. Space Res., 37, 1192-1205, doi: 10.1016/j.asr.2005.03.152, 2006.

Pirjola, R., Kauristie, K., Lappalainen, H., and Viljanen, A.: Space weather risk, Space Weather, 3, S02A02, doi:10.1029/ 2004SW000112, 2005.

Scarpulla, J. and Yarbrough, A.: What Could Go Wrong? The Effects of Ionizing Radiation on Space Electronics, Crosslink, 4, 15-19, 2003.

Sheskin, D. J.: Handbook of Parametric and Nonparametric Statistical Procedures, Chapman \& Hall CRC, 4th edn., 2007.

Simunac, K. D. C. and Armstrong, T. P.: Solar cycle variations in solar and interplanetary ions observed with Interplanetary Monitoring Platform 8, Journal of Geophysical Research (Space Physics), 109, 10101, doi:10.1029/2003JA010194, 2004.

Wilkinson, D.: Trends in environmentally induced spacecraft anomalies, in: NASA/SDIO Space Environmental Effects on Materials Workshop, NASA Conf. Publ. 3035, pp. 123-131, Washington D.C., 1989.

Wilkinson, D. C., Daughtridge, S. C., Stone, J. L., Sauer, H. H., and Darling, P.: TDRS-1 single event upsets and the effect of the space environment, IEEE Transactions on Nuclear Science, 38, 1708-1712, 1991. 